\title{
Etiología y Evaluación del Tratamiento de la Infertilidad en el Instituto Materno Infantil de Bogotá (1982 - 1987)
}

\author{
UNIVERSIDAD NACIONAL DE COLOMBIA \\ FACULTAD DE MEDICINA \\ DEPARTAMENTO DE GINECOLOGIA Y OBSTETRICIA
}

Dres. Carlos Pacheco Consuegra*, Ariel Iván Ruiz Parra**, Jacinto Sánchez Angarita***

La etiología de la infertilidad tiene variaciones regionales e individuales que se reflejan en las diferencias observadas en los informes de la literatura.

El análisis de las publicaciones sobre etiología y tratamiento de la infertilidad se dificulta por la aplicación de diferentes esquemas diagnósticos y terapéuticos entre los diversos centros y aún entre las parejas investigadas en un mismo centro. Otras dificultades estan determinadas por la falta de uniformidad en la forma de diseñar las investigaciones, de presentar los resultados y por tratarse de estudios que involucran dos individuos.

En el presente estudio se analizan los casos de infertilidad atendidos en el Instituto Materno Infantil de Bogotá, identificando los factores causantes, su frecuencia, procedimientos diagnósticos y terapéuticos y se presentan los resultados obtenidos comparándolos con informes de la literatura nacional e internacional.

\section{MATERIALES Y METODOS}

\section{A. DIAGNOSTICO}

Se revisaron 291 historias clínicas de parejas que consultaron a la Clínica de Infertilidad del Departamento de Ginecología y Obstetricia del Instituto Materno Infantil de Bogotá (IMI), durante un período de 54 meses comprendido entre el 1 o. de octubre de 1982 (cuando los autores comienzan sus labores en la Clínica) y el 31 de marzo de 1987. Durante este mismo período se atendieron en la consulta externa de ginecología un total de 14.466 pacientes.

\footnotetext{
* Instructor Asociado.

** Instructor Asociado.

*** Instructor Asociado.
}

Las pacientes fueron remitidas de la consulta externa después de habérseles practicado citología vaginal, laboratorio de rutina y solicitar espermograma al esposo. Antes de iniciar el estudio se realizó una entrevista de información a todas las parejas y posteriormente se elaboró la historia clínica, se practicó el examen físico completo y basado en estos, se orientaron los procedimientos diagnósticos iniciales.

Se investigó anovulación o factor endocrino en aquellas pacientes con irregularidad menstrual, galactorrea, hirsutismo, obesidad, etc. Los estudios comprendían: seguimiento del moco cervical, biopsia endometrial dos días antes de la fecha esperada de la menstruación, determinación del desarrollo folicular y ovulación por ultrasonido (iniciándola el día décimo del ciclo), y dosificaciones de LH, FSH, Estradiol, Progesterona, Prolactina (PRL), TSH, T3, T4, Testosterona y Dehidroepiandrosterona sulfato (DHEAS) en los casos justificados.

Se practicó histerosalpingografía (HSG) y/o laparoscopia en los casos sugestivos de compromiso tuboperitoneal. La laparoscopia se realizó también como examen previo a cirugía tubárica cuando la HSG mostraba obstrucción, sospecha clínica de endometriosis o en ausencia de otros factores que explicaran la infertilidad. La HSG se practicó siempre en las pacientes con historia de aborto habitual.

El diagnóstico de Factor Cervical se estableció cuando se encontró evidencia clínica de cervicitis y cultivo positivo, o lesión orgánica aparente que alteraran la prueba postcoito, anormalidades en la producción de moco cervical en presencia de estimulo estrogénico adecuado y prueba postcoito alterada (con moco cervical y semen adecuados).

Se solicitó un espermograma de control cuando el primero mostró alteraciones de acuerdo con los 
patrones de la OMS (22); ante la persistencia de la alteración se practicó valoración completa por el servicio de urología. El estudio inmunológico se realizó básicamente ante moco hostil, infertilidad inexplicada y en la mayoría de pacientes con alteraciones del factor masculino (test de anticuerpos inmovilizantes).

El cariotipo se solicitó siempre en el estudio del aborto habitual y en la falla ovárica primaria.

\section{B. MANEJO}

La inducción de la ovulación se inició la mayoría de las veces con citrato de clomifeno a dosis de $50 \mathrm{mg} /$ día durante 5 días iniciando el día quinto del ciclo; entre 1982 y 1983 se utilizó ciclofenil a dosis de $600 \mathrm{mg} /$ día con el mismo esquema del clomifeno. En caso de falta de respuesta al citrato de clomifeno, se administraron gonadotropinas (75 MUI de FSH y LH por ampolla) 2 ampollas por vía IM los días sexto, octavo y décimo del ciclo, aunque su uso estuvo limitado en nuestro medio por los costos. La monitoría de la inducción de la ovulación se realizó inicialmente con curva de temperatura basal y puntaje cervical y, desde 1984, con seguimiento del desarrollo folicular por ultrasonido. El uso de gonadotropina coriónica humana (HCG) se orientó de acuerdo con los hallazgos de la monitoría. En los casos de hiperprolactinemia (en ausencia de hipotiroidismo primario y lesión tumoral) se utilizó bromoergocriptina, iniciando con $1.25 \mathrm{mg} /$ día y aumentando la dosis de acuerdo con la respuesta y tolerancia al medicamento. Se administró hormona tiroidea cuando estuvo indicada.

El tratamiento quirúrgico se practicó en aquellas pacientes con compromiso bilateral tuboperitoneal, empleando técnicas microquirúrgicas y usando lupas y microscopio. En aquellas pacientes con compromiso unilateral, el manejo médico inicial se orientó hacia la corrección de los factores asociados. El factor uterino se manejo quirúrgicamente de acuerdo con los hallazgos. En el factor cervical se hizo tratamiento médico. El factor masculino se trato de acuerdo con el compromiso con inseminación heteróloga, inseminación homóloga, citrato de clomifeno, HCG y, en algunos casos, modificación del medio ambiente laboral (exposición al calor, químicos, etc.). Las inseminaciones se practicaron con copa cervical; para las inseminaciones heterólogas se utilizó semen congelado suministrado por el Centro Colombiano de Fertilidad (CECOLFES).
Se clasificaron como esterilidad los casos de falla ovárica primaria y de compromiso tuboperitoneal muy severo, no susceptible de corrección quirúrgica.

\section{RECOLECCION DE LA INFORMACION}

Para este efecto se diseñó un formulario precodificado que incluyó información clínica y paraclínica con el fin de establecer diagnóstico, tratamiento y resultados. Se utilizó en el procesamiento de los datos un computador Apple IIc. Los resultados se presentan en tasas brutas de embarazo, tasas acumulativas de embarazo y no embarazo y tablas de vida y se comparan con los resultados de otras publicaciones. La elaboración de estas tablas se hizo de acuerdo con la metodología descrita por Katayama (6), Cooke (19), Olive (20) y Leridon (21).

\section{RESULTADOS}

\section{A. ETIOLOGIA}

Durante el período de estudio consultaron a la Clínica de Infertilidad del IMI un total de 291 parejas. La edad promedio de las pacientes fue 28.8 años (rango: 17 - 42) y la duración promedio de infertilidad fue de 6.1 años (rango: 1 - 22). La infertilidad primaria se presentó en el $49 \%$ y la secundaria en el $51 \%$.

El promedio de edad para las pacientes con infertilidad primaria fue de 28 años (DS: 5.24 años) con una duración promedio de infertilidad de 5.8 años. En la infertilidad secundaria el promedio de edad fue de 29.5 años (DS: 4.58 años) y el tiempo de exposición fue de 6.3 años.

Se estudió el factor masculino en 213 pacientes encontrándose normal en $164(77 \%)$ y anormal en 49 (23\% ). En el Cuadro No. 1 se especifican las alteraciones encontradas.

El factor tuboperitoneal se estudió en 170 pacientes detectándose anormalidad en 126(74.1\%), con obstrucción bilateral en 77 casos $(61 \%)$ y unilateral en $30(24 \%)$.

De las 126 pacientes con factor tuboperitoneal, $49(38.8 \%)$ tenían infertiliảad primaria y 77 $(61.1 \%)$ secundaria. 
CUADRO No. 1

ESPERMOGRAMA: ANORMALIDADES

\begin{tabular}{|lrr|}
\hline ALTERACION & No. & $\%$ \\
\hline Astenozoospermia & 25 & 51.0 \\
Azoospermia & 14 & 28.7 \\
Líquido seminal anormal $^{*}$ & 7 & 14.3 \\
Teratozoospermia & 1 & 2.0 \\
Aspermia & 1 & 2.0 \\
Anticuerpos & 1 & 2.0 \\
\hline TOTAL & 49 & 100.0 \\
\hline
\end{tabular}

* Incluye: espermatozoides normales con aglutinación o líquido seminal anormal o recuento leucocitario elevado.

CUADRO No. 2

FACTOR TUBOPERITONEAL

\begin{tabular}{|lrr|}
\hline TIPO DE COMPROMISO & No. & $\%$ \\
\hline Obstrucción bilateral & 77 & 61.1 \\
Obstrucción unilateral & 30 & 23.8 \\
Permeable con adherencias & 18 & 14.3 \\
Endometriosis* & 1 & 0.8 \\
\hline TOTAL FACTOR T - P & 126 & 100.0 \\
\hline
\end{tabular}

* Sin adherencias

En 48 pacientes se hizo HSG y laparoscopia encontrándose correlación diagnóstica entre estos dos métodos en $29(68.7 \%)$ y discrepancia en 19 $(31.3 \%)$. Se practicó laparoscopia en 122 pacientes hallándose en 1.0 casos endometriosis leve $(8.2 \%)$.

El factor ovárico se estudió en 163 pacientes demostrándose anovulación en 59 (36.2\%). Se encontraron 7 fallas ováricas primarias. Se dosificó prolactina en 53 pacientes con alteraciones del ciclo encontrándose elevada en 10 (19\%). La Prolactina se determinó en 28 pacientes que presentaban galactorrea encontrándose elevada en 6 $(21.4 \%)$. De 85 pacientes con ciclos menstruales irregulares a quienes se les inició estudio de factor ovárico, se detectó posteriormente factor tuboperitoneal en $29(34.1 \%)$. Se practicaron 21 estudios ecográficos para diagnóstico de ovulación observándose ausencia de desarrollo folicular en 11 (52.4\%), ovulación en $8(38.1 \%)$ y no ruptura folicular en $2(9.5 \%)$.
E1 factor uterino representó el $8.9 \%$ de los factores etiológicos (9 pacientes de 101 estudiadas). Se encontraron 6 miomatosis y 3 malformaciones congénitas. En 6 pacientes en las cuales la HSG sugirió sinequias uterinas, no se lograron confirmar al explorar la cavidad bajo anestesia. Diez cultivos para BK resultaron negativos.

E1 factor cervical correspondió al 11.3\% (33/291). De 107 pruebas postcoito realizadas, se encontraron 6 o más espermatozoides con movilidad grado A en 56 (52.3\%), y en 51 casos la prueba fue insatisfactoria o negativa $(47.7 \%)$.

Se diagnosticó una disfunción sexual (por dos o menos relaciones sexuales al meś) en 17 casos $(10.2 \%)$.

En el Cuadro No. 3 y en la figura No. 1 se muestra la etiología de la infertilidad de nuestras pacientes.

CUADRO No. 3

ETIOLOGIA DE LA INFERTILIDAD

\begin{tabular}{|lr|}
\hline FACTORES & $\%$ \\
\hline Tuboperitoneal & 74.1 \\
Ovárico & 42.9 \\
Masculino & 23.0 \\
Uterino & 14.9 \\
Cervical & 11.3 \\
Disfunción sexual & 10.2 \\
Endometriosis. & 8.1 \\
\hline
\end{tabular}

En el Cuadro No. 4 se comparan los factores etiológicos con otros estudios.

FIGURA 1

ETIOLOGIA DE LA INFERTILIDAD EN EL I.M.I

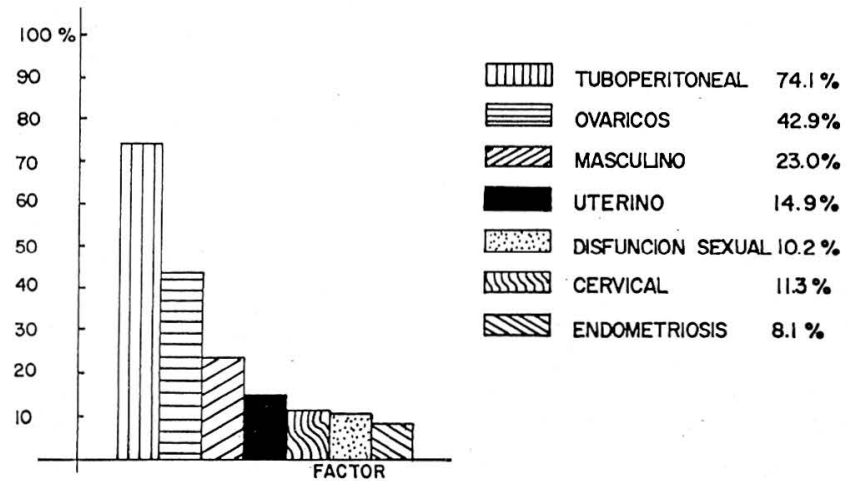


CUADRO No. 4

\section{ETIOLOGIA DE LA INFERTILIDAD}

\begin{tabular}{|c|c|c|c|c|c|c|c|c|}
\hline AUTORES & ANNO & N\& PACENTES & TUBOPERTTONEAL & OVARICO & UTERENO & CERVICAL & ENDOMETRIOSS & Mascurino \\
\hline JONES & 1962 & S5S & 23.6 & 21.8 & 1.8 & 3.6 & 14.7 & 14.3 \\
\hline DOR & 1977 & 665 & 16.3 & 31.5 & 3.8 & 1.1 & & 28 \\
\hline WinKHAUS & 1977 & 2877 & 27.1 & 32.5 & 70 & 80 & & 808 \\
\hline PERALTA C. & 1979 & 232 & 25.6 & is & 20 & 35 & & 209 \\
\hline SANCKEZ F. & 1980 & 202 & 43.6 & 60.4 & 1.5 & 9.4 & 8.0 & 26.2 \\
\hline THOMAS & 1980 & 291 & 11 & 80.2 & & & & 18.8 \\
\hline KLIGEP & 1984 & 493 & 18 & 223 & 1.8 & B.3 & 49 & 20.8 \\
\hline $\begin{array}{l}\text { PRESENTE } \\
\text { ESTUDDOO }\end{array}$ & 1987 & 291 & 74.1 & 42.9 & 14.9 & 11.3 & 8.1 & 23.0 \\
\hline
\end{tabular}

Completaron el estudio 231 parejas; el factor masculino fue el responsable de la infertilidad en el $9.5 \%$, el factor femenino en el $78.8 \%$ y participaron ambos factores en el $11.7 \%$.

\section{B. TRATAMIENTO Y RESULTADOS}

De las 291 parejas $60(20.6 \%)$ abandonaron la consulta apenas iniciado el estudio sin lograrse un diagnóstico; en $118(40.5 \%)$ se estableció el diagnóstico y no se hizo tratamiento (incluyendo 15 casos de esterilidad) y 113 pacientes $(38.8 \%$ ) fueron tratadas una vez hecho el diagnóstico. En el Cuadro No. 5 se analizan los embarazos en estos 3 grupos.

\section{CUADRO No. 5}

\section{RESULTADOS SOBRE EL TOTAL DE PACIENTES}

\begin{tabular}{|c|c|c|c|c|c|}
\hline \multicolumn{2}{|c|}{$\begin{array}{l}\text { GRUPOS DE TOTAL } \\
\text { PACIENTES }\end{array}$} & EMBARAZOS & $\begin{array}{c}\text { TASA DE } \\
\text { EMBARAZOS }\end{array}$ & $\begin{array}{l}\text { EMBARAZOS } \\
\text { A TERMINO } \\
\text { Y EN CURSO }\end{array}$ & $\begin{array}{c}\text { TASA DE } \\
\text { EMBARAZOS } \\
\text { A TERMINO } \\
Y \text { EN CURSO }\end{array}$ \\
\hline DX - TTO & 113 & 46 & 40.7 & 33 & 29.2 \\
\hline DX SIN TTO & 118 & 12 & 10.2 & 8 & 6.8 \\
\hline SIN DX & 60 & 3 & 5 & 2 & 3.3 \\
\hline TOTAL & 291 & 61 & 21.0 & 43 & 14.8 \\
\hline
\end{tabular}

De las 59 pacientes con infertilidad primaria tratada, $23(39 \%)$ lograron el embarazo y de las 54 pacientes con infertilidad secundaria tratada, 23 lograron el embarazo (42.6\%). En el Cuadro No. 6 se relaciona la edad con la tasa de embarazos en las pacientes tratadas.
CUADRO No. 6

CONCEPCIONES POR GRUPOS DE EDAD

\begin{tabular}{|l|cccc|}
\hline & \multicolumn{2}{|c|}{$\begin{array}{r}20 \\
\text { años años }\end{array}$} & $\begin{array}{r}26-30 \\
\text { años }\end{array}$ & $\begin{array}{c}\rangle \\
\text { años }\end{array}$ \\
\hline $\begin{array}{l}\text { No. de casos } \\
\text { Embarazos }\end{array}$ & 2 & 30 & 35 & 46 \\
$\quad$ a término & 1 & 5 & 8 & 8 \\
$\quad \begin{array}{l}\text { en curso } \\
\text { abortos }\end{array}$ & & 5 & 2 & 4 \\
$\quad$ ectópicos & & 2 & 2 & 5 \\
& & & & 3 \\
Total de embarazos & 1 & 12 & 13 & 20 \\
Porcentaje & 50.0 & 40.0 & 37.1 & 43.5 \\
\hline
\end{tabular}

El Cuadro No. 7 relaciona el tiempo de exposición (duración de la infertilidad) con el pronóstico, medido por la tasa de embarazos.

CUADRO No. 7

TASA DE EMBARAZOS SEGUN TIEMPO DE EXPOSICION

\begin{tabular}{|llr|}
\hline AÑOS & EMBARAZOS/CASOS & $\%$ \\
\hline $0-1$ & $4 / 6$ & 66.7 \\
$2-3$ & $16 / 29$ & 55.2 \\
$4-5$ & $10 / 22$ & 45.5 \\
$6-10$ & $14 / 42$ & 33.3 \\
$<10$ & $2 / 14$ & 14.3 \\
\hline
\end{tabular}

Cuando se comparó el grupo de pacientes con menos de 3 años de exposición con aquellas que tenían más de 3 años de exposición se encontró una tasa de embarazos de $57.1 \%$ y $33.3 \%$ respectivamente.

Se practicaron 39 cirugías sobre trompas y lisis de adherencias ocurriendo embarazos intrauterinos en $12(30.8 \%)$ y ectópicos en $2(5.1 \%)$ de estas pacientes hasta la fecha (en estos grupos estan incluidas, 2 pacientes que presentaron un aborto antes de lograr un embarazo de curso normal, una con dos abortos, una con dos ectópicos y una un aborto y un ectópico). Hubo 5 cirugías sobre ovario y paraovario y $3(60 \%)$ de estas pacientes lograron embarazo intrauterino. En 4 pacientes con factor tuboperitoneal (TP) leve se practicaron hidrotubaciones, dos presentaron embarazo intrauterino y una ectópico. En 18 casos de compromiso 
tuboperitoneal unilateral que fueron sometidas a tratamiento para factores asociados, sin tratamiento quirúrgico, ocurrieron 7 embarazos intrauterinos $(38.9 \%)$. En el Cuadro No. 8 se muestra la tasa acumulativa de embarazos en pacientes tratadas para factor tuboperitoneal.

CUADRO No. 8

TASA ACUMULATIVA DE EMBARAZOS POR FACTORES

\begin{tabular}{|cccc|}
\hline MESES & TOTAL & FACTOR T - P & OVARICO \\
\hline 3 & .16 & .12 & .14 \\
6 & .25 & .23 & .20 \\
9 & .35 & .33 & .29 \\
12 & .40 & .38 & .34 \\
15 & .46 & .44 & .40 \\
18 & .51 & .48 & .44 \\
21 & .54 & .53 & .49 \\
24 & .60 & .59 & .54 \\
27 & .60 & .59 & .54 \\
30 & .60 & .59 & .54 \\
33 & .60 & .59 & .54 \\
36 & .60 & .59 & .54 \\
39 & .60 & .59 & .54 \\
\hline
\end{tabular}

Las dos pacientes a quien se les practicó miomectomía quedaron embarazadas.

Se realizó inducción de la ovulación en 61 pacientes lográndose la ovulación en $31(50.8 \%)$ y 19 embarazos $(31 \%$ ) (9 embarazos a término, 3 embarazos en curso, 6 abortos y 1 ectópico). Ginco pacientes recibieron hormona tiroidea, con un embarazo que terminó en aborto; nueve recibieron bromocriptina, de las cuales tres quedaron embarazadas (embarazo a término) y dos pacientes recibieron corticoides lográndose un embarazo que terminó en aborto (esta paciente recibió además inductores de la ovulación). Se hizo monitoría ultrasónica de la inducción de la ovulación en 25 casos. En el Cuadro No. 8 se muestra la tasa acumulativa de embarazos en pacientes tratadas para factor ovárico.

En 17 pacientes $(10.2 \%)$ se encontró en la anamnesis una frecuencia de relaciones sexuales menor de 2/mes; en las dos pacientes en las que este fue el único factor de infertilidad se logró el embarazo al orientar las relaciones sexuales.
Se encontraron 16 parejas (23\%) con compromiso del factor masculino. En 9 pacientes se practicó inseminación homóloga y en 7 inseminación heteróloga. Se lograron 5 embarazos $(31.2 \%)$ en las 16 parejas con factor masculino; 3 fueron el resultado de inseminaciones heterólogas (un embarazo a término, uno en curso y un aborto) mientras que no hubo embarazos en las inseminaciones homólogas.

El factor inmunológico se encontró en una pareja en la que se detectaron anticuerpos antiesperma en ambos cónyuges. Este caso se incluye en el grupo de pacientes no tratadas por retiro voluntario de la clínica. Después de una interrupción de las relaciones sexuales durante 10 meses ocurrió el embarazo; un espermograma de control fue normal.

La tasa bruta de embarazos fue $40.7 \%$ para las pacientes tratadas. Con una tasa de embarazos a término y en curso de $29.2 \%$. Las tasas acumulativas de embarazos por trimestre se muestran en el Cuadro No. 8. La tasa acumulativa máxima $(60 \%)$ se logró a los 24 meses de iniciado el tratamiento. En el Cuadro No. 9 se muestra el análisis de la tabla de vida para todas las pacientes tratadas.

\section{CUADRO No. 9}

ANALISIS DE TABLA DE VIDA PARA PACIENTES TRATADAS POR INFERTILIDAD

\begin{tabular}{|c|c|c|c|c|c|c|c|}
\hline $\begin{array}{l}\text { Intervalo } \\
\text { (meses) }\end{array}$ & $\begin{array}{l}\text { Pacientes no } \\
\text { embarazadas }\end{array}$ & $\begin{array}{l}\text { Número de } \\
\text { embarazos }\end{array}$ & $\begin{array}{l}\text { Deserción } \\
\text { Altas } \\
\text { (D) }\end{array}$ & $\begin{array}{c}\text { Proporción } \\
\text { de } \\
\text { embarazos } \\
\text { (E) }\end{array}$ & $\begin{array}{l}\text { Proporción de } \\
\text { no } \\
\text { embarazos } \\
\text { (F) }\end{array}$ & $\begin{array}{c}\text { Proporción } \\
\text { acumulada } \\
\text { de no } \\
\text { embarazos } \\
\text { (G) }\end{array}$ & $\begin{array}{c}\text { Proporción } \\
\text { acumulada } \\
\text { de } \\
\text { embarazos } \\
\text { (H) }\end{array}$ \\
\hline 3 & 113 & 18 & 22 & .16 & .84 & .84 & .16 \\
\hline 6 & 73 & 8 & 10 & .11 & .89 & .75 & .25 \\
\hline 9 & 55 & 7 & 9 & .13 & .87 & .65 & .35 \\
\hline 12 & 39 & 3 & 5 & .08 & .92 & .60 & .40 \\
\hline 15 & 31 & 3 & 7 & .10 & .90 & .54 & .46 \\
\hline 18 & 21 & 2 & 3 & .10 & .90 & .49 & .51 \\
\hline 21 & 16 & 1 & 1 & .06 & .94 & .46 & .54 \\
\hline 24 & 14 & 2 & 2 & .14 & .86 & .40 & .60 \\
\hline 27 & 10 & 0 & 4. & 0.00 & 1.00 & .40 & .60 \\
\hline 30 & 6 & 0 & 2 & 0.00 & 1.00 & .40 & .60 \\
\hline 33 & 4 & 0 & 1 & 0.00 & 1.00 & .40 & .60 \\
\hline 36 & 3 & 0 & 1 & 0.00 & 1.00 & .40 & .60 \\
\hline 39 & 2 & 0 & 1 & 0.00 & 1.00 & .40 & .60 \\
\hline
\end{tabular}

\section{DISCUSION}

Según Berhman y Kistner, mencionados por Barnea (1), la tasa acumulativa de embarazos en las poblaciones fértiles presenta la siguiente distribución: $25 \%$ al mes de exposición, $63 \%$ a los 6 meses, $75 \%$ a los 9 meses, $80 \%$ a los 12 meses y $90 \%$ a los 18 meses. 
Aproximadamente $19 \%$ de las parejas que inician relaciones sexuales sin anticoncepción, tendrán dificultad para lograr el embarazo al terminar el primer año; de éstas, el $4 \%$ serán estériles y el 15\% subfértiles. Al finalizar el tercer año de exposición al embarazo aproximadamente un 7\% de las parejas no habrá concebido y serán sometidas a procedimientos más complejos de estudio (2).

En el período comprendido entre el 1o. de octubre de 1982 y el 31 de marzo de 1987, asistieron por primera vez a la consulta externa de ginecología del Instituto Materno Infantil de Bogotá, un total de 14.466 pacientes de las cuales 291 consultaron por infertilidad, para una incidencia del $2.1 \%$. Esta cifra no necesariamente refleja la magnitud en la población general dadas las características del hospital, que es una institución de referencia de tercer nivel en la que se atienden pacientes de bajos recursos económicos.

La edad promedio de las pacientes fue de 28.8 años. No hubo diferencia estadísticamente significativa entre las edades de las pacientes con infertilidad primaria y secundaria (p > 0.05) y no se encontró diferencia ( $\mathrm{p}\rangle$ 0.05) en la tasa de embarazos entre las pacientes mayores de 30 años $(43.5 \%)$ y las menores de esa edad $(38.8 \%)$ como ha sido reportado por otros autores que mostraron mejor pronóstico a menor edad de las pacientes $(3,4)$. La duración promedio de la infertilidad fue de 6.1 años. Nuestros hallazgos concuerdan con los de Kliger (3) y Dor (5) en cuanto al mejor pronóstico que tienen las pacientes con menor tiempo de infertilidad; estos autores encontraron una tasa de embarazos de $63 \%$ y $63.1 \%$ (respectivamente) para exposición menor de 3 años comparadas con $33.6 \% y$ $31.6 \%$ cuando es mayor de 3 años; las cifras correspondientes para el presente estudio fueron $57.1 \%$ y $33.3 \%$ (diferencia estadísticamente significativa). Vale la pena señalar que el promedio de duración de la infertilidad de nuestras pacientes fue superior a los 3 años.

No encontramos diferencias significativas entre las tasas de embarazo según el tipo de infertilidad (39\% para la primaria y $42.6 \%$ para la secundaria). Kliger (3) encontró un pronóstico ligeramente mejor en la infertilidad secundaria cuando se comparó con la primaria (con tasas de embarazo del $56.2 \%$ y del $49.7 \%$, respectivamente).

La etiología de la infertilidad en estas pacientes se muestra en el Cuadro No. 3 y en el Cuadro No. 4 , se compara con la de otros estudios, observándo- se la frecuencia más alta de factor tuboperitoneal en nuestra serie ( $75 \%$ aproximadamente). Posibles explicaciones a este hecho son, entre otras, la alta prevalencia de infecciones cervicovaginales y enfermedad pélvica inflamatoria en las pacientes que asisten a nuestro hospital. Cuando se analizó el factor tuboperitoneal, se observó que el 39\% de las pacientes correspondían a infertilidad primaria y el $61 \%$ a secundaria, diferencia estadísticamente significativa $(\mathrm{p}<0.05)$. Llama la atención que la tasa de embarazos de pacientes tratadas para factor ovárico es sensiblemente igual a la de las pacientes tratadas para factor tuboperitoneal, en contraste con otros reportes en los que el factor ovárico tiene mejor pronóstico $(3,5,6)$. Se supone que esta diferencia puede explicarse en parte por el acceso limitado que tienen estas pacientes a la terapéutica con gonadotropinas, cuando están indicadas.

El factor tuboperitoneal se encontró en un $34 \%$ de las pacientes en quienes el estudio se había enfocado inicialmente hacia el factor ovárico por alteraciones obvias en el patrón menstrual; esto reafirma la necesidad de estudiar siempre el factor tuboperitoneal como afirman otros autores (3, 7, 8). Se observó correlación entre las observaciones por laparoscopia e HSG en las dos terceras partes de los casos estudiados por ambos métodos; sin embargo, considerando que la discrepancia en un tercio de los casos incluye un número considerable de pacientes, se sugiere que los dos métodos sean usados en forma complementaria siempre que se encuentre alteración con uno de ellos. La laparoscopia brinda mayor información sobre el factor peritoneal y recomendamos este método como examen inicial.

La endometriosis se presentó sólo en $8.2 \%$ de nuestras pacientes, cifra que contrasta con la de otros informes $(9,23,24)$ que reportan entre 23 y $40 \%$ en pacientes infértiles.

Se encontraron niveles elevados de prolactina en el $21.4 \%$ de las pacientes a quienes se les dosificó por presentar galactorrea y en el $19 \%$ de los casos en los que se determinó por alteraciones del ciclo. Lo que constituye una justificación para determinación de los niveles de prolactina en estos casos. $(10,11)$ (Se encontraron 6 casos de endocrinopatía, todos correspondientes a hipotiroidismo asociado a otros factores).

La frecuencia del factor masculino en esta serie concuerda con la de otros estudios $(5,6)$; el pronóstico en estos casos fue reservado; la tasa de 
embarazos obtenida (31.2\%) se debió en gran parte a las inseminaciones heterólogas en casos de azoospermia. Una observación directa fue frecuencia alta de deserciones en este grupo.

El factor uterino se presentó asociado a otros factores, excepto en dos casos en los que el tratamiento con miomectomía culminó en embarazo. La TBC endometrial no fue investigada rutinariamente en nuestros casos.

El compromiso cervical de tipo infeccioso se observó en un porcentaje alto de pacientes; el factor cervical estuvo generalmente asociado en forma secundaria a otros factores. Solamente en un caso se detectó factor inmunológico que comprometía a ambos miembros de la pareja, al lado de una severa alteración del factor masculino; esta pareja logró el embarazo después de una separación de 10 meses, posiblemente, por mejoría espontánea del factor masculino y de pareja como se ha reportado en otros estudios.

El único tipo de disfunción sexual detectado fue la frecuencia de relaciones sexuales inferior a 2 por mes, en 17 casos. Este dato puede obtenerse fácilmente por la historia clínica y su corrección se logra con la educación a la pareja.

Los 15 casos de estexilidad en este estudio correspondieron a falla ovárica prematura 7, factor tuboperitoneal severo 6, obstrucción tubárica postoperatoria 2 y aborto habitual de causa no detectada 2.

En el Cuadro No. 10 se muestra la tasa bruta de embarazos de esta serie y se compara con otros estudios. Dor (5) cita una tasa de embarazos a término de $28.6 \%$ y de abortos de $7.6 \%$; en nuestro estudio, la tasa de embarazos a término es del $19.5 \%$; si se incluyen los embarazos actualmente en curso es del $29.2 \%$ y la tasa de aborto es del $8.8 \%$.

En la Figura 2, se comparan las tasas acumulativas de embarazos en la población normal en los EEUU según Cooke (19) con las tasas acumulativas de embarazos en pacientes infértiles tratadas por Katayama (6), Lamb (14) y en el presente estudio. Las tasas acumulativas de embarazo son una forma de presentación de resultados que tiene en cuenta el tiempo de seguimiento y la permanencia de las pacientes en el estudio, es superior a las tasas brutas de embarazo pero en ellas se asume que las pacientes que se pierden del seguimiento se comportarían de igual forma que las que continuan en él, lo cual no siempre es cierto. (20) Cuando aplicamos la tasa acumulativa de embarazos a los factores más importantes en este estudio se observó una tasa máxima a los 24 meses (54\% para el factor ovárico y $59 \%$ para el tuboperitoneal). La máxima tasa acumulativa de embarazos para todos los factores fue del $60 \%$ y se logro a los 24 meses.

CUADRO No. 10

TASA BRUTA DE EMBARAZOS

\begin{tabular}{|lll|}
\hline AUTORES & AÑO & TASA (\%) \\
\hline Stone (12) & 1956 & 27 \\
Jones (13) & 1962 & 37.4 \\
Lamb (14) & 1972 & 43 \\
Dor (5) & 1977 & 50.4 \\
Winkhaus (15) & 1977 & 30.3 \\
Peralta (16) & 1979 & 33.7 \\
Katayama (6) & 1979 & 38 \\
Sánchez (17) & 1980 & 25.5 \\
Thomas (18) & 1980 & 50.8 \\
Kliger (3) & 1984 & 52.1 \\
Presente estudio & 1987 & 40.7 \\
\hline
\end{tabular}

FIGURA No. 2

COMPARACION DE TASAS ACUMULATIVAS

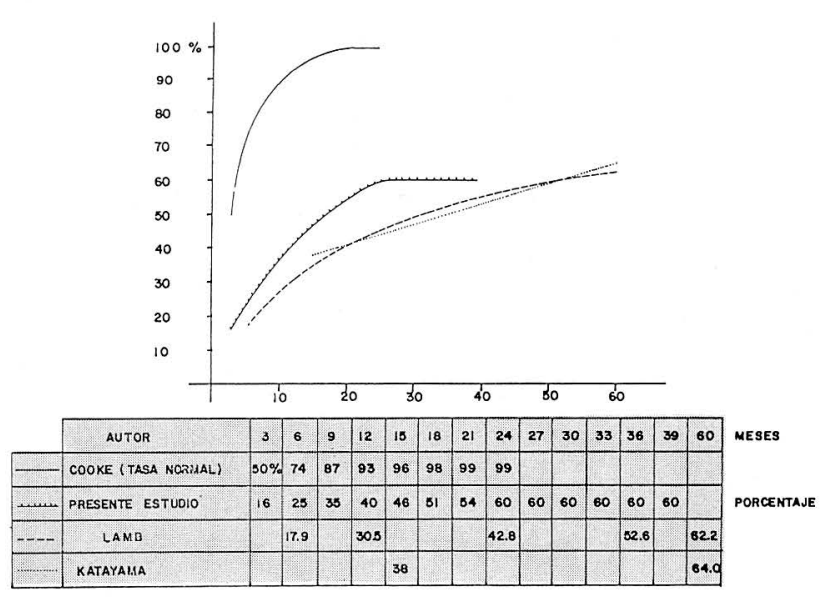




\section{BIBLIOGRAFIA}

1. BARNEA, ER. HOLFORD, TR. McINNES DRA: Long-term prognosis of infertile couples with normal basic investigations: A life-table analysis. Obstet Gynecol 66:24, 1986.

2. SPIRA, A: Epidemiology of human reproduction. Human Reproduction 1:111, 1986.

3. KLIGER, B.E.: Evaluation, therapy, and outcome in 493 infertile couples. Fertil Steril 41:40, 1986.

4. LAMB, E.: Prognosis for the infertile couple. Fertil Steril 23: 320, 1972.

5. DOR, J. HOMBERG, R. RABAU, E: An evaluation of etiologic factors and therapy in 665 infertile couples. Fertil Steril 28:7, 1977.

6. KATAYAMA KP, KAP-SOON JU, MAY M. ET AL: Computer analysis of etiology and pregnancy rate in 636 cases of primary infertility. Am J. Obstet Gynecol 135: 207, 1979.

7. HUTCHINS C J.: Laparoscopy and hysterosalpingography in the assessment of tubal patency. Obstet Gynecol 49: 325, 1977.

8. SWOLIN K, ROSENCRANTZ M: Laparoscopy vs. hysterosalpingography in sterility investigations: a comparative study. Fertil Steril 23: 270, 1972.

9. COHEN M: Diagnóstico laparoscópico y tratamiento seudomenopáusico de la endometriosis con danazol. Clin.Obstet.Ginecol. 3: 919, 1980.

10. QUIGLEY M.M., HANEY AF: Valoración de la hiperprolactinemia: perfiles clínicos. Clin. Obstet. Ginecol. 2: 333, 1980.

11. BLANKSTEIN J, MASHIACH S, LUNENFELD, B: Ovulation induction and in vitro fertilization. Year Book Medical Publishers, Inc. Chicago-London, 1986. pp. 211.

12. STONE, A., and WARD, M.E.: Factors responsible for pregnancy in 500 infertility cases, Fertil Steril 7: $1,1956$.
13. JONES, A. and POURMAND K: An evaluation of etiologic factors and therapy in 555 private patients with primary infertility. Fertil Steril 13:5, 1962.

14. LAMB EJ. CRUZ AL: Data collection and analysis in an infertility practice. Fertil Steril 23:310, 1972.

15. WINKHAUS I: Esterilidad de la pareja mono o multifactorial. Distribución de frecuencia y resultados del tratamiento. En "Reproducción Humana. Fertilidad, esterilidad y contracepción". pp. 314 Ed. Salvat, 1985.

16. PERALTA M.T. REVOLLO PF, GARCIA C F.: Estudio de la pareja esteril en el Hospital Universitario de San Ignacio. Rev. Col. Obst.Gin. 30: 1979.

17. SANCHEZ E F., DEVIA M G., HENAO CG, et al: Embarazos en 223 pacientes tratadas por esterilidad durante un período de 5 años. Rev. Col. Obstet. Ginecol.33: 183, 1981.

18. THOMAS AK, FORREST M S.: Infertility: A review of 291 infertile couples over eight years. Fertil Steril 34: $106,1980$.

19. COOKE ID, SULAIMAN RA, LENTON E.A. et al: Fertility and infertility statistics: their importance and application. Clin. Obstet. Gynecol 8: 531, 1981.

20. OLIVE DL.: Analysis of clinical fertility trials: a methodologic review. Fertyl Steryl 45: 157, 1986.

21. LERIDON H, SPIRA A: Problems in measuring the effectiveness of infertility therapy. Fertyl Steryl 41: 580, 1984.

22. WHO: Simplified management of the infertile couple. Study 84914. 1986.

23. WORLEY R J.: Endometriosis. Serono Symposia, August 1982: 80.

24. STILLMAN R J.: Endometriosis in the infertile female. Serono Symposia, August 1983: 103. 